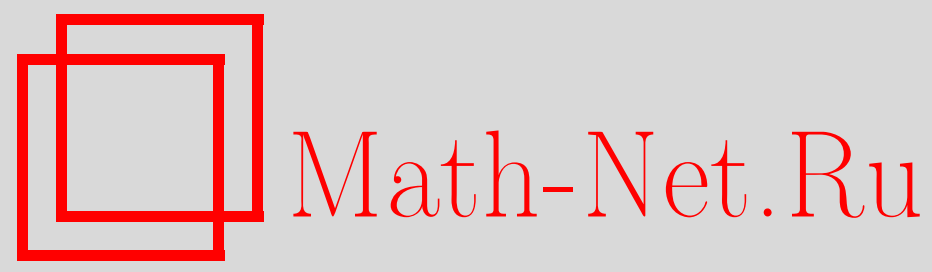

А. И. Штерн, Жесткость и аппроксимация квазипредставлений аменабельных групп, Матем. заметки, 1999, том 65, выпуск 6, 908-920

DOI: https://doi.org/10.4213/mzm1126

Использование Общероссийского математического портала Math-Net.Ru подразумевает, что вы прочитали и согласны с пользовательским соглашением http://www.mathnet.ru/rus/agreement

Параметры загрузки:

IP : 54.237 .59 .107

26 апреля 2023 г., 13:30:06

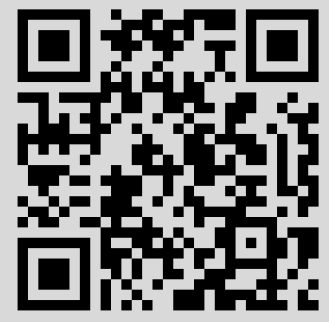




\title{
ЖЕСТКОСТЬ И АППРОКСИМАЦИЯ КВАЗИПРЕДСТАВЛЕНИЙ АМЕНАБЕЛЬНЫХ ГРУПП
}

\section{А. И. Штерн}

\begin{abstract}
Получены достаточные условия того, что (необязательно ограниченное) квазипредставление аменабельной (вообще говоря, топологческой) группы есть ограниченное возмущение обычного представления. В частности, показано, что произвольное (необязательно ограниченное) конечномерное квазипредставление аменабельной топологической группы есть ограниченное возмущение обычного представления.

Библиографой: 27 названий.
\end{abstract}

Введение. Квазигомоморфизм $T$ полугрупш $S$ в равномерную полугрупп $\Sigma-$ это отображение $T: S \rightarrow \Sigma$ такое, что элементы $T\left(s_{1} s_{2}\right)$ и $T\left(s_{1}\right) T\left(s_{2}\right), s_{1}, s_{2} \in S$, равномерно близки. Изучение таких отображений представляет естественньй интерес, связанный с объединением идей симметрии и близости (соответствующая задача была поставлена в связи с результатами статей [1] и [2] еще в книге Улама [3]), но оно имеет и некоторьй физический смысл, которьй можно описать следующим образом. Если у группы $G$, являющейся группой симметрий описания некоторой физической системы, существуют квазипредставления (т.е. квазигомоморфизмы в группу обратимых непрерьвных линейных операторов в некотором топологическом векторном пространстве), для которых величина окружения $U$ определяется точностью измерений и не существует “достаточно близких" обычных представлений группы $G$ в том же топологическом векторном пространстве, то интерпретация эксперимента может оказаться более сложной, чем в случае, когда близкое представление действительно существует, и это может потребовать тщательного различения истинных симметрий (связанных с “законами природы") и квазисимметрий.

Условия существования гомоморфизмов, близких к данному квазигомоморфизму группы или полугрупшы, изучаются с различных точек зрения более 50 лет [4]-[12], [1], [2], [13]-[22]; примеры квазипредставлений, не допускаюших близких представлений, рассматривались в [7]-[10], [15]-[17], [19]-[26].

В настоящей статье получены достаточные условия того, что (необязательно ограниченное) квазипредставление аменабельной (вообе говоря, топологической) группы есть ограниченное возмущение обычного представления. В частности, доказано, что

Работа выполнена при частичной поддержке Российского фонда фундаментальных исследований, грант № 96-01-00276, программы “Ведущие научные школы", грант № 96-15-96249, и фонда DFG, грант "Indextheorie", FA.-№ 04030206. 
для любого (вообще говоря, неограниченного) конечномерного квазипредставления аменабельной грушы существует обычное представление, ограниченным возмущением которого является исходное квазипредставление, и изучаются условия существования близких представлений такого типа при условии, что нормы $\left\|T\left(s_{1} s_{2}\right)-T\left(s_{1}\right) T\left(s_{2}\right)\right\|$, $s_{1}, s_{2} \in G$, равномерно малы.

Работа построена следующим образом. В п. 1 получены некоторые общие результаты о свойствах квазипредстравлений с неограниченными орбитами и, в частности, показано, что $\varepsilon$-квазипредстравление $T$ аменабельной группы $G$ в сопряженном банаховом пространстве с $\varepsilon<1 / 2$, удовлетворяющее дополнительным условиям $T(e)=1$ и $T(g)^{-1}=T\left(g^{-1}\right), g \in G$, является $\varphi(\varepsilon)$-возмущением обычного представления, где $\varphi(\varepsilon) \rightarrow 0$ при $\varepsilon \rightarrow+0$. В п. 2 определяется структура конечномерных квазипредставлений аменабельных групп.

Часть результатов п. 2 опубликована без доказательств в [26].

1. Квазипредставления с неограниченными орбитами. Приведем сначала определения и основные свойства.

ОПРЕДЕЛЕНИЕ 1.1 [17], [21]. Пусть $S$ - полугруппа, $E$ - топологическое векторное пространство. Отображение $T$ полугрупшы $S$ в алгебру $L(E)$ непрерывных линейных операторов в $E$ называется квазипредставлением (точнее, $U$-квазипредставлением, где $U$ - данное равностепенно непрерывное семейство в $L(E))$ полугрупшы $S$ в $E$, если семейство $U(T)$, определяемое равенством

$$
U(T)=\left\{T\left(s_{1} s_{2}\right)-T\left(s_{1}\right) T\left(s_{2}\right), s_{1}, s_{2} \in S\right\}
$$

равностепенно непрерьвно (соответственно, если $U(T)$ содержится в $U$ ).

В нормированном пространстве $\varepsilon$-квазипредставление $(\varepsilon \geqslant 0)$ есть $U$-квазипредставление с $U=\{T, T \in L(E),\|T\|<\varepsilon\}$.

Очевидно, что любое $\varepsilon$-квазипредставление является квазипредставлением, а 0-квазипредставление есть обычное представление.

Один из наиболее общих вопросов, связанных с $\varepsilon$-квазипредставлениями, состоит в изучении условий, обеспечивающих существование обычного представления полугруппы, близкого к данному квазипредставлению, и изучение свойств множества этих представлений, если оно непусто. В статьях [10], [13], [27] (другие ссылки можно найти в библиографии к этим статьям) изучался вопрос о существовании близких представлений для случая, когда полугруппа $S$ есть компактная группа (в статье [12] получены условия тривиальности одномерных отображений, близких к аддитивньм, структура которых в общем случае изучена в [19], [21]). Кроме того, в статье [10] рассматривался также случай $\varepsilon$-квазипредставлений аменабельных локально компактных групп, однако, как отмечено в статье [23], это исследование содержит неточность, связанную с тем, что если к непрерывной функции двух переменных (пробегающих аменабельную группу) применить инвариантное среднее по одному из этих переменньг, то может получиться разрьвная функция другого переменного (и даже функция, не являющаяся борелевской, см. [18]); напомним, что пробел в доказательстве соответствующего утверждения в классе сильно непрерьвных квазипредставлений заполнен в [21] с помощью результата статьи [9].

Напомним следующее 
ОПРЕДЕЛЕНИЕ 1.2 [17], [21]. Пусть $G$ - аменабельная локально компактная группа, $B$ - банахово пространство. Группа $G$ называется устойчиво представимой в банаховом пространстве $B$, если для любых $\delta>0$ и $C>0$ существует $\varepsilon>0$ такое, что если $T$ - сильно непрерывное $\varepsilon$-квазипредставление групш $G$ в $B$ обратимыми операторами, удовлетворяющее условиям

$$
\|T(g)\| \leqslant C, \quad\left\|T(g)^{-1}\right\| \leqslant C
$$

для всех $g \in G$, то существует представление $R$ грушшы $G$ в $B^{*}$ такое, что $\left\|T(g)^{*}-R(g)\right\| \leqslant \varepsilon$ для всех $g \in G$ и матричные элементы $g \mapsto(R(g) F)(x), g \in G$, непрерывны при $F \in B^{*}, x \in B$.

Как известно, справедлива

ТЕОРемА 1.1 [17], [21]. Любая аменабельная локально компактная группа устойчиво представима в любом банаховом пространстве.

Отметим очевидное следствие этой теоремы.

СлЕДСТВИЕ 1.1 [17], [21]. Пусть $G$ - группа, В - банахово пространство. Для любого $C>0$ и $\varepsilon>0$ существует $\delta>0$ такое, что для любого $\delta$-квазипредставления $T$ группы $G$ в $B$ обратимыми операторами, удовлетворяющего условиям

$$
\|T(g)\| \leqslant C, \quad\left\|T(g)^{-1}\right\| \leqslant C
$$

для всех $g \in G$, существует (ограниченное) $\varepsilon$-квазипредставление $R$ группь $G$ в $B$, удовлетворяющее условиям $R(e)=1 u\|T(g)-R(g)\| \leqslant \varepsilon д_{\text {я }}$ всех $g \in G$.

Если $G$ - топологическая группа и отображение $T$ сильно непрерывно, то (при надлежащем выборе $\varepsilon$ ) квазипредставление $R$ можно выбрать также сильно непрерывным.

Это утверждение решает общую задачу для ограниченных квазипредставлений и мотивирует следующую гипотезу.

ГИПОТЕЗА. Любое сильно непрерывное квазипредставление аменабельной локально компактной группы является ограниченным возмущением обычного непрерывного представления әтой группы.

Структура важного класса неограниченных квазипредставлений, а именно, квазипредставлений, замькания орбит которых содержат нулевой элемент [17], [21], также подкрепляет эту гипотезу. Приведем типичное утверждение.

ТеОрема 1.2 [17], [21]. Пусть E - топологическое векторное пространство, $G$ - группа, $T$ - квазипредставление группь $G$ непрерывными линейными операторами в пространстве Е. Предположсим, что операторы квазипредставления $T$ обратимы и существует ненулевой әлемент $x \in E$ такой, что замыкание $\overline{O_{T^{-1}}(x)}$ соответствующей $T^{-1}$-орбиты $O_{T^{-1}}(x)=\left\{T(g)^{-1} x, g \in G\right\}$ әлемента $x \in E$ содержит нулевой элемент пространства $E$. Тогда замкнутая линейная оболочка $F$ орбиты $O_{T^{-1}}(x)$ инвариантна относительно $T$ и ограничение отображения $T$ на $F$ есть обычное представление группы $G$.

Легко видеть, что любое квазипредставление обратимыми операторами, для которого замыкание $\overline{O_{T^{-1}}(x)}$ некоторой ненулевой $T^{-1}$-орбиты содержит нулевой элемент 
пространства $E$, непременно неограничено. Оказывается, все неограниченные квазипредставления имеют специфические свойства.

Введем следующие обозначения. Если $E$ - сопряженное банахово пространство, то мы обозначим через $E_{*}$ некоторое банахово пространство, к которому сопряжено пространство $E$. Кроме того, если $G$ - аменабельная локально компактная группа, то мы обозначим через $I$ некоторое двусторонне инвариантное среднее на $L^{\infty}(G)$ (если подьнтегральная функция зависит от нескольких переменных, то в индексе указьвается переменная, по которой берется среднее). Если $M: G \rightarrow L(E)$ - ограниченная функция со значениями в непрерьвных линейных операторах в пространстве $E$, то мы определим непрерьвньй линейный оператор $I(M)$ в пространстве $E$ формулой

$$
(I(M) x)(f)=I_{g}((M(g) x)(f)), \quad x \in E, \quad f \in E_{*} .
$$

Лемма 1.1. Пусть $G$ - группа, $T$ - ее неограниченное квазипредставление в банаховом пространстве $E=E_{T}$.

1) Квазипредставление $T$ имеет хотя бы одну неограниченную орбиту.

2) Для любих $g, h \in G u x \in E$ T-орбита вектора $(T(g h)-T(g) T(h))$ х ограничена. В частности, если все ненулевые $T$-орбиты неограничены, то $T$ - обычное представление.

3) Пусть $L$ - векторное подпространство $E$, образованное всеми әлементами $x \in E$ такими, что орбита $O_{T}(x)=\{T(g) x, g \in G\}$ ограничена. Снабдим $L$ нормой

$$
\|x\|_{L}=\sup _{g \in G}\|T(g) x\|, \quad x \in E, \quad \text { əдe }\|\cdot\|=\|\cdot\|_{E}
$$

Тогда тохдественное отобрахение $j: L \rightarrow E$ непрерывно $u L$ полно относительно нормы $\|\cdot\|_{L}$. Векторное подпространство $L \subset E$ инвариантно относительно всех операторов $T(g), g \in G$, и соотношения

$$
\begin{aligned}
\|T(g) x\|_{L} & \leqslant\|x\|_{L}+\varepsilon\|x\|, \\
\|(T(k) T(l)-T(k l)) x\|_{L} & \leqslant \varepsilon\|T(l) x\|+2 \varepsilon\|x\|, \quad g, k, l \in G, \quad x \in E,
\end{aligned}
$$

выполняются для всех $g, k, l \in G ;$ в частности, $\|T(k) T(l)-T(k l)\|_{L} \leqslant 3 \varepsilon$ для всех $k, l \in G$.

4) Пусть $S$ - квазипредставление группь $G$, являющееся ограниченным возмущением квазипредставления $T$, т.е. отображсение $S-T: G \rightarrow \mathscr{L}(E)$ имеет ограниченный образ. Тогда образ оператора $S(g)-T(g)$ содержится в подпространстве $L \subset E$ для любого $g \in G$. В частности, если все ненулевые $T$-орбить в $E$ неограничены (и $T$ - обычное представление, см. утвержсдение 2)), то $T$ жестко в том смысле, что если ограниченное возмущение $S$ квазипредставления $T$ является квазипредставлением, то $S$ совпадает с самим $T$.

ДокАЗАТЕЛЬСтво. Утверждение 1) непосредственно следует из принципа равномерной ограниченности.

Рассмотрим утверждение 3 ). Очевидно, что множество $L$, введенное в формулировке, действительно является векторным подпространством в $E$ (так как суммы и кратные 
ограниченных множеств ограничены), a $\|\cdot\|_{L}$ действительно является нормой на $L$. Непрерьвность отображения $j: L \rightarrow E$ также очевидна, поскольку

$$
\|x\|_{L}=\sup _{g \in G}\|T(g) x\| \geqslant\|x\|_{E}, \quad x \in E .
$$

Если $\left\{x_{n}\right\}, x_{n} \in L$, фундаментальна в $L$, то для любого $\varepsilon>0$ существует натуральное $N$ такое, что $\left\|T(g) x_{n}-T(g) x_{m}\right\|_{E} \leqslant \varepsilon$ для всех $g \in G$ и всех $n, m>N$. Следовательно, для любого $g \in G$ существует $y(g) \in E$ такой, что $\left\|T(g) x_{n}-y(g)\right\| \leqslant \varepsilon$ для всех $n>N$. Однако, $T(g) x_{n} \rightarrow T(g) x$, где $x=\lim x_{n}$ (напомним, что если $\left\{x_{n}\right\}$ фундаментальна в $L$, то она фундаментальна и в $E)$. Поэтому $\left\|T(g) x_{n}-T(g) x\right\| \leqslant \varepsilon$ для всех $n>N$, или $\left\|x_{n}-x\right\|_{L} \leqslant \varepsilon$ при $n>N$, что доказьвает полноту $L$.

Для доказательства первого соотношения в (1.2) воспользуемся основным неравенством $\|T(h) T(k)-T(h k)\| \leqslant \varepsilon$, или $\|T(h) T(k) x-T(h k) x\| \leqslant \varepsilon\|x\|$. Если $x \in L$, то отсюда сразу следует, что

$$
\|T(h)(T(k) x)\| \leqslant\|T(h k) x\|+\varepsilon\|x\| \leqslant\|x\|_{L}+\varepsilon\|x\| \leqslant(1+\varepsilon)\|x\|_{L},
$$

что доказьвает одновременно и инвариантность $L$ относительно $T(h)$ для любого $h \in G$, и первое соотношение в (1.2).

Для доказательства второго соотношения в (1.2) рассмотрим тождество

$$
\begin{aligned}
T(g)(T(k) T(l)-T(k l))= & (T(g) T(k)-T(g k)) T(l)+(T(g k) T(l)-T(g k l)) \\
& +(T(g k l)-T(g) T(k l)) .
\end{aligned}
$$

Применяя его к $x \in E$ и вычисляя норму, получаем, что

$$
\|T(g)(T(k) T(l)-T(k l)) x\| \leqslant \varepsilon\|T(l) x\|+2 \varepsilon\|x\| .
$$

По определению нормы в $L$ отсюда следует, что

$$
\|(T(k) T(l)-T(k l)) x\|_{L} \leqslant \varepsilon\|x\|_{L}+2 \varepsilon\|x\| .
$$

Это доказьвает второе соотношение в (1.2).

Заметим теперь, что, в частности, второе соотношение в (1.2) доказьвает первую часть утверждения 2), а вторая часть утверждения 2) сразу следует из первой части этого утверждения.

Рассмотрим утверждение 4). Пусть $S$ - квазипредставление групшы $G$, являющееся ограниченньм возмущением квазипредставления $T, R=T-S$ ограничено. Тогда $T$ и $S$ - квазипредставления, $R$ ограничено и множество $\{T(g h)-T(g) T(h)=S(g h)+$ $R(g h)-S(g) S(h)-S(g) R(h)-R(g) S(h)-R(g) R(h), g, h \in G\}$ ограничено. Отсюда следует ограниченность $\{S(g) R(h)+R(g) S(h), g, h \in G\}$. При любом фиксированном $h \in G$ это означает, что $\{S(g) R(h) x, g \in G\}$ является ограниченным множеством для любого $x \in E$. Таким образом, $R(h) x \in L$ для всех $g \in G$ и $x \in E$. Это завершает доказательство леммы.

Приведем достаточные условия того, что данное квазипредставление аменабельной дискретной групшы есть ограниченное (и даже малое) возмущение обычного представления. 
ТеОрема 1.3. Пусть $G$ - аменабельная дискретная группа и $T$ - (вообще говоря, неограниченное) $\delta$-квазипредставление группь $G$ в сопряженном банаховом пространстве $E, \delta<1 / 2$. Предположим дополнительно, что $T(e)=1_{E}$ $u T(g)^{-1}=T\left(g^{-1}\right)$ для всех $g \in G$. Существует (обичное) представление $S$ группь $G$ в $E$ такое, ито $\|S(g)-T(g)\| \leqslant \varepsilon$ для всех $g \in G$, где $\varepsilon=f(\delta)$ c $f(t)=1 /(2-4 t)$, $t \in[0,1 / 2)$.

ДокАЗАтЕЛьСтво. Так как $T-\delta$-квазипредставление, для всех $g, h \in G$ имеем $\left\|T(g h) T\left(h^{-1}\right)-T(g)\right\| \leqslant \delta$. В частности, $h \mapsto T(g h) T\left(h^{-1}\right)$ - ограниченная операторнозначная функция. Положим $S(g)=I_{h}\left(T(g h) T\left(h^{-1}\right)\right)$ для всех $g \in G$. Ввиду двусторонней инвариантности среднего $I$ имеем

$$
\begin{aligned}
S(g)= & I_{h}\left(T(g h) T\left(h^{-1}\right)\right)=I_{h}\left(T(g k h) T\left((k h)^{-1}\right)\right)=I_{h}\left(T(g k h) T\left(h^{-1} k^{-1}\right)\right) \\
= & I_{h}\left((T(g k h)-T(g k) T(h))\left(T\left(h^{-1} k^{-1}\right)-T\left(h^{-1}\right) T\left(k^{-1}\right)\right)\right. \\
& \left.+T(g k) T(h)\left(T\left(h^{-1} k^{-1}\right)-T\left(h^{-1}\right) T\left(k^{-1}\right)\right)+T(g k h) T\left(h^{-1}\right) T\left(k^{-1}\right)\right) \\
= & \Delta+T(g k) I_{h}\left(T(h)\left(T\left(h^{-1} k^{-1}\right)-T\left(h^{-1}\right) T\left(k^{-1}\right)\right)\right)+S(g k) T\left(k^{-1}\right)
\end{aligned}
$$

для всех $g, k \in G$, где через $\Delta$ обозначен оператор

$$
I_{h}\left((T(g k h)-T(g k) T(h))\left(T\left(h^{-1} k^{-1}\right)-T\left(h^{-1}\right) T\left(k^{-1}\right)\right)\right),
$$

норма которого не превосходит $\delta^{2}$. Из соотношения $T(h)^{-1}=T\left(h^{-1}\right)$, где $h \in G$, следует, что

$$
I_{h}\left(T(h)\left(T\left(h^{-1} k^{-1}\right)-T\left(h^{-1}\right) T\left(k^{-1}\right)\right)\right)=I_{h}\left(T(h) T\left(h^{-1} k^{-1}\right)\right)-T\left(k^{-1}\right),
$$

а из инвариантности среднего (с помошью замены $h \mapsto k l$ и перехода к среднему по $l$ ) заключаем, что

$$
I_{h}\left(T(h) T\left(h^{-1} k^{-1}\right)\right)-T\left(k^{-1}\right)=I_{l}\left(T\left(k^{-1} l\right) T(l)\right)-T\left(k^{-1}\right)=S\left(k^{-1}\right)-T\left(k^{-1}\right) .
$$

Получаем, что

$$
S(g)=\Delta+T(g k)\left(S\left(k^{-1}\right)-T\left(k^{-1}\right)\right)+S(g k) T\left(k^{-1}\right),
$$

или

$$
T(g k) T\left(k^{-1}\right)-T(g k) S\left(k^{-1}\right)-S(g k) T\left(k^{-1}\right)+S(g)=\Delta,
$$

для всех $g, k \in G$. С другой стороны, имеем

$$
\begin{aligned}
(S(g k)-T(g k))\left(S\left(k^{-1}\right)-T\left(k^{-1}\right)\right)= & S(g k) S\left(k^{-1}\right)-T(g k) S\left(k^{-1}\right) \\
& -S(g k) T\left(k^{-1}\right)+T(g k) T\left(k^{-1}\right)
\end{aligned}
$$

для всех $g, k \in G$, и можно вычесть последнее тождество из предыдущего. Получаем, что

$$
S(g)-S(g k) S\left(k^{-1}\right)=\Delta-(S(g k)-T(g k))\left(S\left(k^{-1}\right)-T\left(k^{-1}\right)\right),
$$


откуда $\left\|S(g)-S(g k) S\left(k^{-1}\right)\right\| \leqslant 2 \delta^{2}$, поэтому $S$ является $2 \delta^{2}$-квазипредставлением группы $G$, причем $\|S(g)-T(g)\| \leqslant \delta$. Положим $T=T_{n}$ и $S=T_{n+1}$. Тогда при $\delta=\delta_{1}<1 / 2$ оценка

$$
\delta_{n+1} \leqslant 2 \delta_{n}^{2} \leqslant 2^{2^{n+1}-1} \delta_{1}^{2^{n+1}}=\frac{1}{2}\left(2 \delta_{1}\right)^{2^{n+1}}
$$

немедленно приводит к требуемому результату для отображения, определяемого как предел (по отношению к равномерной сходимости на группе в смысле нормы оператора) $\widetilde{T}=\lim _{n \rightarrow \infty} T_{n}$, которое и оказьвается искомьм 0-квазипредставлением, или обычным представлением.

Заметим, что приведенное выше доказательство частично использует идею метода Ньютона, использованную при установлении основного результата статьи [9] в форме, переработанной в соответствии с [23].

Следующий результат, в формулировке которого не предполагается, что квазипредставление $T$ удовлетворяет дополнительньм условиям $T(e)=1_{E}$ и $T(g)^{-1}=T\left(g^{-1}\right)$ для всех $g \in G$, может потребовать большей точности в оценке величины $\| T(g) T(h)-$ $T(g h) \|, g, h \in G$, но относится к непрерьвным квазипредставлениям произвольной аменабельной локально компактной группы. Кажется правдоподобным, что представление группы в банаховом пространстве, для которого образ некоторой малой окрестности единищы этой групшы лежит в малом открытом шаре с центром в единичном операторе, автоматически непрерывно, но доказательство этого утверждения автору неизвестно. Поэтому в формулировке следующей теоремы есть предположение о непрерывности представления в пространстве $L$, которое может оказаться излишним.

ТЕОремА 1.4. Пусть $G$ - аменабельная локально компактная группа и $T$ - (вообще говоря, неограниченное) $\delta$-квазипредставление группы $G$ в сопряженном банаховом пространстве Е. Предполохим дополнительно, что существует (обычное) непрерывное представление $S_{L}$ группь $G$ в $L$, для которого оператор $J_{L}=$ $I\left(\left.S_{L}\left(h^{-1}\right) T(h)\right|_{L}\right)$ корректно определен, непрерывен и непрерывно обратим. Тогда существует (обычное) представление $S$ группы $G$ в $E$ такое, что $\| S(g)-$ $T(g) \| \leqslant C$ для всех $g \in G$ и некоторого $C$.

ЗАмЕчАниЕ. Пусть $G$ - аменабельная локально компактная группа и $\delta$-квазипредставление $T$ в банаховом пространстве $E$ сопряжено к (вообще говоря, неограниченному) $\delta$-квазипредставлению групшы $G$ в банаховом пространстве $E_{*}$, где $\delta<1 / 60$. Предположим дополнительно, что квазипредставление, действующее в подпространстве $L$, непрерьвно, а $L$ изоморфно сопряженному банахову пространству (см. утверждение 3 ) леммы 1.1), что выполняется автоматически, если $L$ замкнуто в $E$, а $E$ рефлексивно. Тогда существует (обычное) непрерывное представление $S_{L}$ групшы $G$ в $L$, для которого оператор $J_{L}=I\left(\left.S_{L}\left(h^{-1}\right) T(h)\right|_{L}\right)$ корректно определен, непрерьвен и непрерьвно обратим, и существует (обычное) представление $S$ группы $G$ в $E$ такое, что $\|S(g)-T(g)\| \leqslant \varepsilon$ для всех $g \in G$, где $\varepsilon=f(\delta)$ с $f(t)=2 t(1+3 t) /\left(1-2(1+3 t)\left(4+8(1+t)^{2}\right) t\right), t \in[0,1 / 60)$.

ДОКАЗАТЕЛЬСТВО ЗАМЕЧАНИЯ. В условиях замечания имеем

$$
\begin{aligned}
\|T(g)\|_{L} & \leqslant 1+\delta, \\
\|(T(k) T(l)-T(k l)) x\|_{L} & \leqslant \delta\|T(l) x\|+2 \delta\|x\|, \quad g, k, l \in G, \quad x \in E
\end{aligned}
$$

(см. (1.2)), поэтому $\|T(k) T(l)-T(k l)\|_{L} \leqslant 3 \delta$ и применение следствия из теоремы Джонсона [9], полученного в [17] (см. также [21]), показьвает, что существует непрерьвное 
представление $S_{L}$ грушшы $G$ в $L$, удовлетворяюшее условиям $\left\|S_{L}(g)\right\|_{L} \leqslant 2(1+\delta), g \in G$, и $\left\|S_{L}-\left.T\right|_{L}\right\| \leqslant \lambda$, где $\lambda=\left(4+8(1+3 \delta)^{2}\right) 3 \delta$. Заметим, что $\sigma=2(1+3 \delta) \lambda<1$ при $\delta<1 / 60$. Отсюда следует, что

$$
\left\|1_{L}-\left.S_{L}\left(h^{-1}\right) T(h)\right|_{L}\right\| \leqslant \sigma<1,
$$

тогда и $\left\|1-J_{L}\right\| \leqslant \sigma<1$, где $J_{L}=I\left(\left.S_{L}\left(h^{-1}\right) T(h)\right|_{L}\right)$, так что оператор $J_{L}$ обратим и $\left\|J_{L}^{-1}\right\| \leqslant 1 /(1-\sigma)$. Положим $\varepsilon=2(1+3 \delta) \delta /(1-\sigma)$. Дальнейшее рассуждение относится к доказательству теоремы 1.4 .

ДоКАЗАТЕЛЬСТВО ТЕОРЕМЫ 1.4. Положим

$$
B_{L}(k)=I_{h}\left(S_{L}\left(h^{-1}\right)(T(h k)-T(h) T(k))\right) \in \mathscr{L}(E, L)
$$

(т.е. этот оператор рассматривается как оператор из $E$ в $L$; напомним, что образ оператора $T(h k)-T(h) T(k)$ содержится в $L$ для любых $h, k \in G)$. Заметим, что $\left\|B_{L}(k)\right\| \leqslant$ $C \delta$, если $\left\|S_{L}(k)\right\| \leqslant C$. Тогда

$$
\begin{aligned}
B_{L}(k l)= & I_{h}\left(S_{L}\left(h^{-1}\right)(T(h k l)-T(h) T(k l))\right) \\
= & I_{h}\left(S_{L}\left(h^{-1}\right)(T(h k l)-T(h k) T(l))\right)+I_{h}\left(S_{L}\left(h^{-1}\right)(T(h k)-T(h) T(k) T(l))\right) \\
& +I_{h}\left(S_{L}\left(h^{-1}\right) T(h)(T(k l)-T(k) T(l))\right) \\
= & S_{L}(k) B_{L}(l)+B_{L}(k) T(l)+J_{L}(T(k l)-T(k) T(l))
\end{aligned}
$$

(мы использовали замену $h k \mapsto w$ и усреднение по $w$ в первом слагаемом).

Положим $J_{L}^{-1} B_{L}(k)=C_{L}(k)$ и $J_{L}^{-1} S_{L}(k) J_{L}=\sigma(k), k \in G$; в частности, $\sigma$ - тоже представление групшы $G$ в $L$. Так как

$$
j C_{L}(k l)=j \sigma_{L}(k) C_{L}(l)+j C_{L}(k) T(l)+(T(k l)-T(k) T(l)) \in \mathscr{L}(E)
$$

ввиду (1.5), то

$$
T(k l)-T(k) T(l)+j C_{L}(k l)-j \sigma_{L}(k) C_{L}(l)-j C_{L}(k) T(l)=0 .
$$

Из определения $B_{L}$ следует, что на $L$ выполняется соотношение

$$
\begin{aligned}
\left.B_{L}(k)\right|_{L} & =I_{h}\left(\left.S_{L}\left(h^{-1}\right)(T(h k)-T(h) T(k))\right|_{L}\right) \\
& =I_{h}\left(\left.S_{L}\left(h^{-1}\right) T(h k)\right|_{L}\right)-I_{h}\left(S_{L}\left(h^{-1}\right) T(h)\right) T(k) .
\end{aligned}
$$

Мы легко получаем, что на $L$ выполняется соотношение

$$
\left.B_{L}(k)\right|_{L}=\sigma_{L}(k) J_{L}-\left.J_{L} T(k)\right|_{L}, \quad k \in G,
$$

где использована замена $h k \mapsto l$ и усреднение по $l$. Тогда для $C_{L}$ имеем $\left.C_{L}(k)\right|_{L}=$ $\sigma_{L}(k)-\left.T(k)\right|_{L}, k \in G$, и для $T^{\prime}(g)=T(g)+j C_{L}(g), g \in G$, мы получаем окончательную формулу

$$
\begin{aligned}
T^{\prime}(k l)-T^{\prime}(k) T^{\prime}(l)= & T(k l)-T(k) T(l)+j C_{L}(k l)-j C_{L}(k) T(l) \\
& -j C_{L}(k) C_{L}(l)-T(k) j C_{L}(l) \\
= & j \sigma_{L}(k) C_{L}(l)-T(k) j C_{L}(l)-j C_{L}(k) C_{L}(l) \\
= & j\left(\sigma_{L}(k)-\left.T(k)\right|_{L}-C_{L}(k)\right) C_{L}(l)=0
\end{aligned}
$$

(в вычислении использовано соотношение $T(k) j C_{L}(l)=j T(k) C_{L}(l), k, l \in G$, связанное с тем, что образ $C_{L}(l)$ содержится в $L$, а $L$ является $T(k)$-инвариантным). По построению $T^{\prime}-T$ есть ограниченное отображение. Это завершает доказательство теоремы. 


\section{2. Структура конечномерных квазипредставлений аменабельных групп.} В этом пункте гипотеза, сформулированная в п. 1, доказьвается для всех конечномерных квазипредставлений аменабельных групп. Напомним структуру произвольного конечномерного квазипредставления.

Tеорема 2.1 [17], [21]. Пусть $G$ - әруппа, $T$ - квазипредставление группь $G$ в конечномерном векторном пространстве $E_{T}$. Пусть $E_{T}^{*}-$ пространство, сопряженное $\kappa E_{T}$. Пусть $L$ - множество векторов $\xi \in E_{T}$ таких, что орбита $\{T(g) \xi, g \in G\}$ ограничена; $M$ - множество функиионалов $f \in E_{T}^{*}$ таких, что орбита $\left\{T(g)^{*} f, g \in G\right\}$ ограничена в $E_{T}^{*}$. Тогда $L$ и аннулятор $M^{\perp}$ подпространства $M$ - векторные подпространства в $E_{T}$, инвариантные относительно T. Рассмотрим направленный по возрастанию набор подпространств $\{0\}$, $L \cap M^{\perp}, M^{\perp}, L+M^{\perp}, E=E_{T}$ u запишем матрииу $t(g)$ оператора $T(g), g \in G, \boldsymbol{\theta}$ блочной форме, отвечающей разложсению пространства $E$ в прямую сумму подпространств $L \cap M^{\perp}, M^{\perp} \backslash\left(L \cap M^{\perp}\right), L \backslash\left(L \cap M^{\perp}\right)$ u $E \backslash\left(L+M^{\perp}\right)$, где “ \" означает взятие дополнительного подпространства, а базис выбран так, чтобы первые две группы его әлементов последовательно образовывали базисы в подпространствах $L \cap M^{\perp}$ и $M^{\perp} \backslash\left(L \cap M^{\perp}\right)$, а следуюшие две группы әлементов образовывали базисы в $L \backslash\left(L \cap M^{\perp}\right)$ и $E \backslash\left(L+M^{\perp}\right)$, причем соответствующие әлементы биортогонального базиса принадлежсли подпространству $M$ пространства $E_{T}^{*}$ :

$$
t(g)=\left(\begin{array}{cccc}
\alpha(g) & \varphi(g) & \sigma(g) & \tau(g) \\
0 & \beta(g) & 0 & \rho(g) \\
0 & 0 & \gamma(g) & \chi(g) \\
0 & 0 & 0 & \delta(g)
\end{array}\right), \quad g \in G
$$

(Здесь $t_{23}(g)=0$, так как $L$ инвариантно относительно T.) Тогда справедливь следующие утверждения:

1) отобразсения $\alpha, \delta, \gamma, \sigma, \chi$ ограничены;

2) отображсения $t_{1}$ u $t_{2}$, определяемые равенствами

$$
t_{1}(g)=\left(\begin{array}{cc}
\alpha(g) & \varphi(g) \\
0 & \beta(g)
\end{array}\right), \quad t_{2}(g)=\left(\begin{array}{cc}
\beta(g) & \rho(g) \\
0 & \delta(g)
\end{array}\right)
$$

являются представлениями группы $G$;

3) отображение является квазикоциклом относительно представлений $t_{1} u$ $t_{2}$, т.е. отобрахсение

$$
(g, h) \mapsto \tau(g h)-\alpha(g) \tau(h)-\varphi(g) \rho(h)-\tau(g) \delta(h), \quad g, h \in G,
$$

ограничено.

ЗАмЕчАниЕ 2.1. Если отображение $\tau$ ограничено на некотором векторе $\xi$ из пространства представления $\delta$, то из формулы (2.3) следует, что отображение $(x, y) \mapsto$ $\varphi(x) \rho(y) \xi$ ограничено по $y \in G$ (при фиксированном $x \in G$ ). Тогда можно показать, что общее ядро семейства $\varphi(x), x \in G$, нетривиально. 
ЗАмечАниЕ 2.2. Рассмотрим отображение вида

$$
T(g)=\left(\begin{array}{cc}
1_{E} & \tau(g) \\
0 & 1_{F}
\end{array}\right), \quad g \in G
$$

в прямой сумме линейных пространств $E+F$, где $\tau(g)$ - непрерывньй линейньй оператор из $F$ в $E$. Такое отображение $T$ называется аддитивным квазипредставлением [21], если семейство операторов

$$
\left\{\tau\left(g_{1} g_{2}\right)-\tau\left(g_{1}\right)-\tau\left(g_{2}\right), g_{1}, g_{2} \in G\right\}
$$

ограничено в совокупности.

Сформулируем основной результат этого пункта.

ТЕОРема 2.2. Пусть $G$ - аменабельная топологческая группа.

1) Любое конечномерное $\varepsilon$-квазипредставление группь $G$ есть ограниченное возмущение обичного представления.

2) Радиус шара, содержащ,го все операторы этого ограниченного возмущ,ния, определяется числом в и нормами ограниченных компонент квазипредставления, упомянутыми в теореме 2.1 .

ДокАЗАТЕЛЬСТво. Из теоремы 2.1 непосредственно следует, что отображения $t_{3}$ и $t_{4}$, определенные формулами

$$
t_{3}(g)=\left(\begin{array}{cc}
\alpha(g) & \sigma(g) \\
0 & \gamma(g)
\end{array}\right), \quad t_{4}(g)=\left(\begin{array}{cc}
\gamma(g) & \chi(g) \\
0 & \delta(g)
\end{array}\right), \quad g \in G
$$

ограничены и являются квазипредставлениями аменабельной группы $G$. Поэтому (по теореме 1.2) достаточно проверить оба утверждения теоремы для квазикоцикла $\tau$. Так как компоненты $\sigma$ и $\chi$ ограничены, при этой проверке мы вправе считать их нулевыми (поскольку замена их нулями есть ограниченное возмущение исходного отображения). Так как компонента $\gamma$ ограничена, мы вправе считать еепредставлением (по теореме 1.1; впрочем, и замена отображения $\gamma$ на отображение в единичньй оператор в подпространстве $L \backslash\left(L \cap M^{\perp}\right)$ есть ограниченное возмущение исходного отображения $\left.t\right)$. Если подпространство $L \cap M^{\perp}$ нульмерно, то из перечисленных вьше свойств отображения $t$ непосредственно следует, что отображение $t$ есть ограниченное возмущение обычного представления. Фактически, достаточно установить, что $\tau$ есть ограниченное возмущение соответствующего $\left\{t_{1}, t_{2}\right\}$-коцикла в предположении, что исходное отображение имеет блочньй вид:

$$
t(g)=\left(\begin{array}{cccc}
\alpha(g) & \varphi(g) & 0 & \tau(g) \\
0 & \beta(g) & 0 & \rho(g) \\
0 & 0 & \gamma(g) & 0 \\
0 & 0 & 0 & \delta(g)
\end{array}\right), \quad g \in G
$$

Пусть $I$ - некоторое правоинвариантное среднее на $G$. Применим $I$ к правой части (2.3), умноженной слева на ограниченную величину $\alpha(g)^{-1}=\alpha\left(g^{-1}\right), g \in G$. Итак, если мы введем величину $\psi$ с помощю формулы

$$
\psi(h)=I_{g}\left(\left(\alpha(g)^{-1}\right)(\tau(g h)-\varphi(g) \rho(h)-\tau(g) \delta(h))\right), \quad h \in G
$$


(напомним, что это среднее имеет смысл, поскольку в (2.6) функция под знаком среднего равномерно ограничена на групе $G$ ), то разность $\tau(g)-\psi(g), g \in G$, будет ограниченной величиной (оцениваемой через $\varepsilon$ и норму $\alpha$ ). Тогда остается проверить, что $\psi-$ коцикл относительно представлений $t_{1}$ и $t_{2}$, т.е. что

$$
\psi(g h)=\alpha(g) \psi(h)+\varphi(g) \rho(h)+\tau(g) \delta(h), \quad g, h \in G
$$

(cp. $(2.3))$.

Чтобы проверить (2.7), найдем левую часть:

$$
\psi(g h)=I_{k}\left(\left(\alpha(k)^{-1}\right)(\tau(k g h)-\varphi(k) \rho(g h)-\tau(k) \delta(g h))\right), \quad g, h \in G,
$$

и воспользуемся тем, что ввиду правой инвариантности $I$ справедлива формула

$$
\psi(h)=I_{k}\left(\left(\alpha(k g)^{-1}\right)(\tau(k g h)-\varphi(k g) \rho(h)-\tau(k g) \delta(h))\right), \quad g, h \in G .
$$

Вычитая (2.9) из (2.8), умноженного слева на $\alpha(g)^{-1}$, и учитывая то, что $\alpha$ - (обычное) представление, мы находим

$$
\begin{aligned}
\alpha(g)^{-1} \psi(g h)-\psi(h)= & I_{k}\left(\left(\alpha(k g)^{-1}\right)(\varphi(k g) \rho(h)+\tau(k g) \delta(h)\right. \\
& -\varphi(k) \rho(g h)-\tau(k) \delta(g h))), \quad g, h \in G .
\end{aligned}
$$

Замечая, что $t_{1}$ и $t_{2}-$ представления групшы $G$, и подставляя в $(2.10)$ соответствующие выражения для функций от $g h$, получаем равенство

$$
\begin{aligned}
\alpha(g)^{-1} \psi(g h)-\psi(h)= & I_{k}\left(\left(\alpha(k g)^{-1}\right)((\varphi(k) \beta(g)+\alpha(k) \varphi(g)) \rho(h)+\tau(k g) \delta(h)\right. \\
& -\varphi(k)(\rho(g) \delta(h)+\beta(g) \rho(h))-\tau(k) \delta(g) \delta(h))), \quad g, h \in G .
\end{aligned}
$$

Перегруппируем слагаемые в правой части (2.10), выделяя в качестве сомножителя под знаком среднего группу слагаемых $\tau(k g)-\varphi(k) \rho(g)-\tau(k) \delta(g)$ :

$$
\begin{aligned}
\alpha(g)^{-1} & \psi(g h)-\psi(h) \\
& =I_{k}\left(\alpha(k g)^{-1} \varphi(k) \beta(g) \rho(h)+\alpha\left(g^{-1}\right) \varphi(g) \rho(h)\right. \\
& \left.+\alpha(k g)^{-1}(\tau(k g)-\varphi(k) \rho(g)-\tau(k) \delta(g)) \delta(h)-\alpha(k g)^{-1} \varphi(k) \beta(g) \rho(h)\right) \\
& =I_{k}\left(\alpha\left(g^{-1}\right) \varphi(g) \rho(h)+\alpha(k g)^{-1}(\tau(k g)-\varphi(k) \rho(g)-\tau(k) \delta(g)) \delta(h)\right) \\
& =\alpha\left(g^{-1}\right) \varphi(g) \rho(h)+I_{k}\left(\alpha(k g)^{-1}(\tau(k g)-\varphi(k) \rho(g)-\tau(k) \delta(g)) \delta(h)\right), \quad g, h \in G .
\end{aligned}
$$

Первое слагаемое в правой части (2.12) совпадает с первым слагаемым в (2.7) с точностью до введенного вьше множителя $\alpha(g)^{-1}$. При вычислении второго множителя воспользуемся тем, что $\alpha$ - представление, и перестановочностью операции взятия операторного инвариантного среднего с умножением слева и справа на линейные операторы, не зависящие от параметра, по которому берется среднее:

$$
\begin{aligned}
& I_{k}\left(\alpha(k g)^{-1}(\tau(k g)-\varphi(k) \rho(g)-\tau(k) \delta(g)) \delta(h)\right) \\
& \quad=I_{k}\left(\alpha(g)^{-1} \alpha(k)^{-1}(\tau(k g)-\varphi(k) \rho(g)-\tau(k) \delta(g)) \delta(h)\right) \\
& \quad=\alpha(g)^{-1} I_{k}\left(\alpha(k)^{-1}(\tau(k g)-\varphi(k) \rho(g)-\tau(k) \delta(g))\right) \delta(h) \\
& \quad=\alpha(g)^{-1} \psi(g) \delta(h), \quad g, h \in G,
\end{aligned}
$$

г де использовано определение отображения $\psi$ с помощью (2.6). Эта величина совпадает со вторым слагаемым в (2.7) с точностью до введенного вьше множителя $\alpha(g)^{-1}$, что завершает доказательство формулы (2.7) и, тем самым, доказывает теорему 2.2 . 
ПримеР. Если в условиях замечания 2.2 положить $E=F=\mathbb{C}$ и взять в качестве $G$ группу $\operatorname{SL}(2, \mathbb{Z})$, а в качестве $\tau$ - псевдохарактер Радемахера, введенньй в [16], то полученное двумерное квазипредставление групшы $G$ не является ограниченным возмушением никакого обычного двумерного представления этой групш. Таким образом, для представлений размерности $\geqslant 2$ условие аменабельности групшы существенно. Для одномерных представлений это не так, и любое одномерное квазипредставление любой групшы есть ограниченное возмущение обычного (одномерного) представления (если это квазипредставление ограничено; если же данное одномерное квазипредставление неограничено, то оно само является обычным одномерным представлением), см. [14] и приведенную вьше теорему 2.1 .

\section{СПИСОК ЦИТИРОВАННОЙ ЛИТЕРАТУРЫ}

[1] Hyers D. H. On the stability of the linear functional equation // Proc. Nat. Acad. Sci. USA. 1941. V. 27. № 2. P. 222-224.

[2] Hyers D. H., Ulam S. M. On approximate isometry // Bull. Amer. Math. Soc. 1945. V. 51. P. 288-292.

[3] Улам С. Нерешенные математические задачи. М.: Наука, 1964.

[4] Baker J., Lawrence J., Zorzitto F. The stability of equation $f(x y)=f(x) f(y) / /$ Proc. Amer. Math. Soc. 1979. V. 74. № 2. P. 242-246.

[5] Gelfand I. Zur Theorie der Charactere der Abelschen topologischen Gruppen // Матем. сб. 1941. T. 9(51). №1. C. 49-50.

[6] Grove K., Karcher H., Roh E.A. Jacobi fields and Finsler metrics on a compact Lie groups with an application to differential pinching problems // Math. Ann. 1974. V. 211. № 1. P. 7-21.

[7] Johnson B. E. Approximately multiplicative functionals // J. London Math. Soc. 1986. V. 34. № 3. P. 489-510.

[8] Johnson B. E. Continuity of generalised homomorphisms // Bull. London Math. Soc. 1987. V. 19. №1. P. $67-71$.

[9] Johnson B. E. Approximately multiplicative maps between Banach algebras // J. London Math. Soc. 1988. V. 37. № 2. P. 294-316.

[10] Kazhdan D. On $\varepsilon$-representations // Israel J. Math. 1982. V. 43. № 4. P. 315-323.

[11] Lawrence J. W. The stability of multiplicative semigroup homomorphisms to real normed algebras // Aequationes Math. 1985. V. 28. №1-2. P. 94-101.

[12] Forti G. L. The stability of homomorphisms and amenability, with applications to functional equations // Abh. Math. Sem. Univ. Hamburg. 1987. V. 57. P. 215-226.

[13] Censer D. The stability problem for transformations of the circle // Proc. Roy. Soc. Edinburgh. Sect. A. 1979. V. 84. P. 279-281.

[14] Штерн А. И. Об устойчивости гомоморфизмов в группу $R^{*} / /$ Вестн. МГУ. Сер. 1. Матем. , мех. 1982. № 3. С. 29-32.

[15] Штерн А.И. Устойчивость представлений и псевдохарактеры // Ломоносовские чтения. М.: МГУ, 1983.

[16] Штерн А. И. Псевдохарактер, определенный символом Радемахера // УМН. 1990. Т. 45. № 3. C. 197-198.

[17] Штерн А. И. Квазипредставления и псевдопредставления // Функцион. анализ и его прилож. 1991. Т. 25. № 2. С. 87-91.

[18] Штерн А.И. О жесткости положительных характеров // УМН. 1980. Т. 35. № 5. С. 218.

[19] Штерн А. И. Непрерывные псевдохарактеры на связных локально компактных группах являются характерами // Функцион. анализ и его прилож. 1993. Т. 27. №4. С. 94-96.

[20] Штерн А. И. Характеризации аменабельных групп в классе связных локально компактных групп // УМН. 1994. Т. 49. № 2. С. 183-184.

[21] Shtern A. I. Pseudosymmetry. I // Russian J. Math. Phys. 1994. V. 2. №3. P. 353-382. 
[22] Штерн А.И. Quasisymmetry and almost representations // Международная конференция "Классическая и квантовая геометрия однородных пространств" (Москва, 12-20 августа 1994). Тезисы докл. М.: Центр “Софус Ли”, 1994.

[23] Файзиев В.А. Псевдохарактеры на свободных произведениях полугрупп // Функцион. анализ и его прилож. 1987. Т. 21. №1. С. 86-87.

[24] Файзиев В. А. Псевдохарактеры на свободных группах и некоторых групповых конструкциях // УМН. 1988. Т. 43. №5. С. 225-226.

[25] Shtern A. I. Almost reresentations and quasi-symmetry // Lie Groups and Lie Algebras. Their Representations, Generalizations and Applications / ed. B.P. Komrakov et al. Math. Appl. (Soviet Ser.). V. 433. Dordrecht-Boston-London: Kluwer Acad. Publ., 1998. P. 337-358.

[26] Shtern A. I. Triviality and Continuity of Pseudocharacters and Pseudorepresentations // Russian J. Math. Phys. 1997. V. 5. № 1. P. 135-138.

[27] de la Harpe P., Karoubi M. Representations approchées d'un groupe dans une algébre de Banach // Manuscripta Math. 1977. V. 22. № 3. P. 297-310.

Московский государственный университет им. М. В. Ломоносова

Поступило

04.02 .96

Исправленный вариант

09.10 .98 